'Departamento de Cirugía Digestiva. Escuela de Medicina de la Pontificia Universidad Católica de Chile. Santiago, Chile.

${ }^{2}$ Departamento de Medicina Interna, Grupo Medicina Hospitalaria. Escuela de Medicina de la Pontificia Universidad Católica de Chile. Santiago, Chile. anterno de Medicina. Escuela de Medicina de la Pontificia Universidad Católica de Chile. Santiago, Chile.

Los autores no refieren conflictos de intereses.

Trabajo no recibió financiamiento.

Recibido el 9 de mayo de 2020 aceptado el 13 de octubre de 2020.

Correspondencia a:

Dr. Gonzalo Eymin Marcoleta 367, Quinto Piso, Medicina. Santiago, Chile. geymin@med.puc.cl

\section{Resección quirúrgica de tapón de cianoacrilato variceal infectado posterior a esclerosis endoscópica de várices gástricas. Caso clínico}

\author{
EDUARDO BRICEÑO ${ }^{1}$, GREGORIO MATURANA ${ }^{1}$, \\ MARÍA J. IRARRÁZAVAL ${ }^{\mathrm{a}}$, SEBASTIÁN LARRAÍN ${ }^{\mathrm{a}}$, \\ GONZALO EYMIN²
}

\section{Removal of an infected gastric cyanoacrylate plug. Report of one case}

In patients with actively bleeding gastric varices, the treatment of choice is the endoscopic use of sclerosing agents such as cyanoacrylate. We report a 69-year-old man who, after being treated with cyanoacrylate, suffered from recurrent febrile episodes. After an extensive study and broad-spectrum antibiotic treatment, discarding other presumably infectious focus, the superinfection of the cyanoacrylate plug was suspected, and its surgical removal was decided. A partial gastrectomy of the gastric fundus, a splenectomy, and a distal pancreatectomy were performed. The patient evolved without fever and without new episodes of bacteremia, but with decompensation of his cirrhosis manifested by ascites, spontaneous bacterial peritonitis, pneumonia, and collections in the pancreatic bed. These complications were managed with medical treatment consisting in a long course of broad-spectrum antibiotics. Thereafter, the patient evolved satisfactorily.

(Rev Med Chile 2020; 148: 1674-1678)

Key words: Cyanoacrylates; Esophageal and Gastric Varices; Infections; Sclerotherapy.
T na de las complicaciones más graves de la hipertensión portal es la hemorragia digestiva secundaria a la ruptura de várices esofágicas o gástricas. La prevalencia de várices gástricas en pacientes con hipertensión portal varía entre $18 \%$ y $70 \%{ }^{1}$. Asociado a su sangrado, se describe una tasa de mortalidad de $45 \%{ }^{1}$. Frente a pacientes con várices gástricas sangrando activamente, el tratamiento de elección es endoscópico, siendo de primera línea el uso de agentes esclerosantes como el cianocrilato, el cual, al contactar la sangre, se polimeriza formando un molde plástico que controla el sangrado. Sin embargo, su uso puede tener complicaciones, las que fluctuan entre
$0,5 \%$ y $5 \%^{2}$ y que incluyen tromboembolismo pulmonar, trombosis portal y mesentérica, sepsis persistente, fistulización, erosión de la mucosa debido a inyección extravascular y extrusión tardía o ulceración de várices. Dentro de estas, la infección del tapón de cianoacrilato (coloquialmente: "histoacriloma"), y la sepsis persistente asociada, es una de las más infrecuentes. Se presenta el caso de un paciente que presentó un síndrome febril prolongado secundario a la infección del tapón variceal de cianoacrilato y que requirió, luego de prolongado tratamiento antibiótico y extensa evaluación, una resección quirúrgica de este para su tratamiento definitivo. 


\section{Caso clínico}

Hombre de 69 años, con antecedentes de diabetes mellitus tipo II no insulinorrequirente. Consultó al servicio de urgencias por caída de altura de 2 metros, resultando con contusión torácica izquierda. Se realizó una tomografía axial computada (TAC) donde se describieron fracturas entre el $7^{\circ}$ y $10^{\circ}$ arco costal posterior izquierdo, hemotórax leve y signos de daño hepático crónico (DHC). Durante la hospitalización presentó una hemorragia digestiva alta con compromiso hemodinámico. Se realizó una endoscopía digestiva alta, en la que se observa abundante sangre en estómago y esófago, várices esofágicas grandes con puntos rojos y sangrado activo, las que se trataron con ligadura endoscópica. El control a las $72 \mathrm{~h}$ no mostraba sangrado y se objetivó adecuada ligadura variceal. El paciente fue dado de alta y, a las 4 semanas, en el control endoscópico se observaron várices esofágicas grandes con puntos rojos y en fondo gástrico una várice gástrica prominente (GOV2). Se realizó nueva ligadura de várices esofágicas e inyección de cianoacrilato más lipiodol en várice gástrica. Volvió a consultar a las 2 semanas por cuadro febril de $24 \mathrm{~h}$ de evolución asociado a calofríos y hematuria. En sus exámenes destacaba orina inflamatoria con glóbulos blancos $(+++)$ y bacterias $(+++)$ y se hospitalizó con diagnóstico de prostatitis. Hemocultivos y urocultivo fueron positivos para Escherichia coli. El paciente persistió febril pese a tratamiento antibiótico, por lo que se estudió nuevamente con imágenes, destacando en TAC un derrame pleural izquierdo loculado y en la resonancia nuclear magnética (RNM) de pelvis, prostatitis, sin abscesos prostáticos. Evaluado por cirugía torácica se indicó manejo médico. Permaneció febril con episodios de repercusión hemodinámica por 3 semanas, por lo que se escaló terapia antibiótica y se estudió con ecocardiograma transtorácico que descartó endocarditis infecciosa. Tras reevaluación periódica por el equipo de cirugía torácica se determinó realizar una videotoracoscopía (4 semanas posterior a la inyección de cianoacrilato). Se realizó decorticación e instalación de drenaje pleural. Los cultivos fueron positivos para Streptococcus sanguinis $y$ Staphylococcus warnerii, quedando en tratamiento con cefuroximo y metronidazol. Sin embargo, persistió con alzas febriles y calofríos con episodios de repercusión hemodinámica, algunos de ellos con hemocultivos positivos para Escherichia coli multisensible, que requirió cambio de antibióticos, iniciando ceftriaxona e imipenem ante persistencia de los episodios. Tras 6 semanas de iniciado el cuadro febril, se estudió con PET-CT con FDG (tomografía de emisión de positrones con fluoro-deoxiglucosa) en el que se observó aumento de metabolismo/captación en área esofagogástrica, hallazgos que se repitieron en nuevo PET-CT con FDG realizado a las 10 semanas (Figura 1). Dada la sospecha de sobreinfección del tapón variceal de cianocrilato y falta de respuesta al tratamiento antibiótico de amplio espectro, se decidió, junto con medicina interna, hepatología, infectología y cirugía digestiva realizar una laparotomía exploradora. En la intervención se observaron grandes paquetes varicosos en región subcardial e "histoacriloma" con proceso inflamatorio que se originaba en el fondo gástrico y se extendía al hilio esplénico, cola de páncreas y vena esplénica. (Figura 2). Se realizó una gastrectomía parcial de fondo gástrico, esplenectomía y resección de paquetes varicosos trombosados con cianoacrilato en su interior, algunos de ellos en contacto estrecho con cola de páncreas, requiriendo, además, una pancreatectomía distal (Figura 3 y 4 ). El paciente evolucionó afebril, con descompensación de su DHC con ascitis a tensión, peritonitis bacteriana espontánea y neumonía. Se trató con terapia depletiva, antibióticos y albúmina. Por persistencia de parámetros inflamatorios elevados se controló con TAC, el cual mostró colecciones en relación al lecho pancreático, las que fueron tratadas con imipenem, dada pobre respuesta a piperacilina/ tazobactam. Evolucionó afebril, hemodinámicamente estable y con resolución de la ascitis. Fue dado de alta 4 semanas posterior a la cirugía con amoxicilina/calvulánico más ciprofloxacino. La biopsia de la pieza operatoria confirmó los signos de trombosis variceal con un grave proceso inflamatorio crónico y granulomatoso en los tejidos adyacentes.

A los 2 meses reingresó por un cuadro febril sin foco. El ecocardiograma transesofágico no mostraba vegetaciones y los hemocultivos fueron negativos. Dado antecedente de portación de Klebsiella productora de carbapenemasas y ante la mala respuesta a imipenem se decidió dejar con ceftazidima avibactam. La TAC de control a las 2 semanas mostró un importante descenso del tamaño de las colecciones, además, con mejoría 


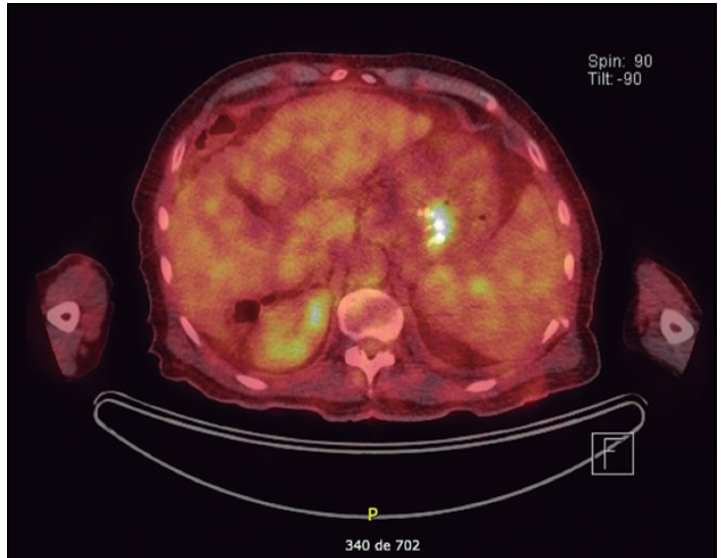

Figura 1. PET-CT FDG a las 10 semanas desde el inicio del cuadro febril. Se observan signos de circulación colateral porto-sistémica en ligamento gastrohepático y presencia de material de escleroterapia en várices del fondo gástrico. En el material de escleroterapia (flecha azul) se reconoce hipermetabolismo glucídico focal (SUV máximo: 5,2 - 6,3).

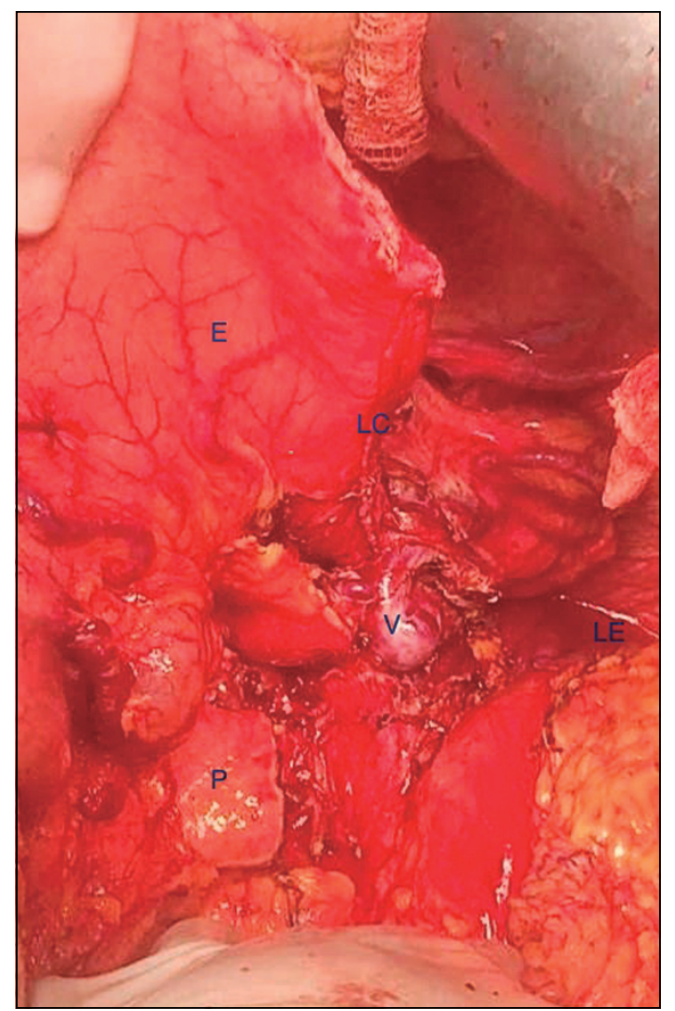

Figura 3. Fotografía intraoperatoria posterior a la resección de la pieza operatoria que muestra la línea de corchetes de sección gástrica (LC), cuerpo de páncreas $(P)$ y logia esplénica (LE). V: várices de pared posterior gástrica, E: estómago.

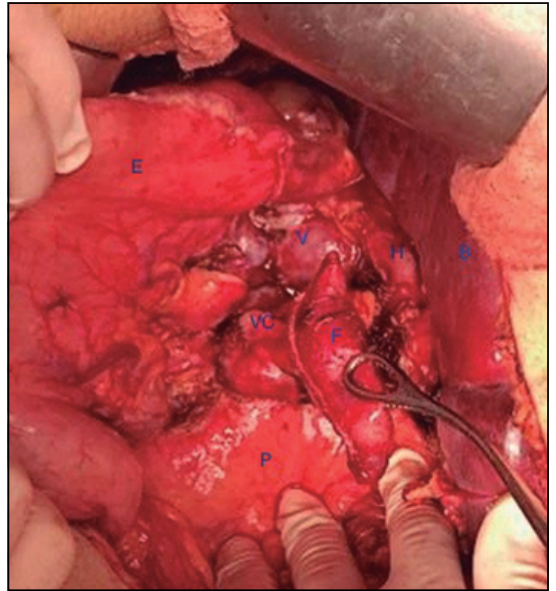

Figura 2. Fotografía intraoperatoria que muestra várices gástricas de pared posterior $(V)$. Se observa un segmento de fondo gástrico (F) ya seccionado desde el estómago (E), unido aún a un extenso proceso inflamatorio en relación a várices gástricas con cianoacrilato en su interior (VC) y que se extiende hasta el hilio esplénico $(H)$ y cola de páncreas $(P)$. Bazo (B) con marcado aumento de tamaño.

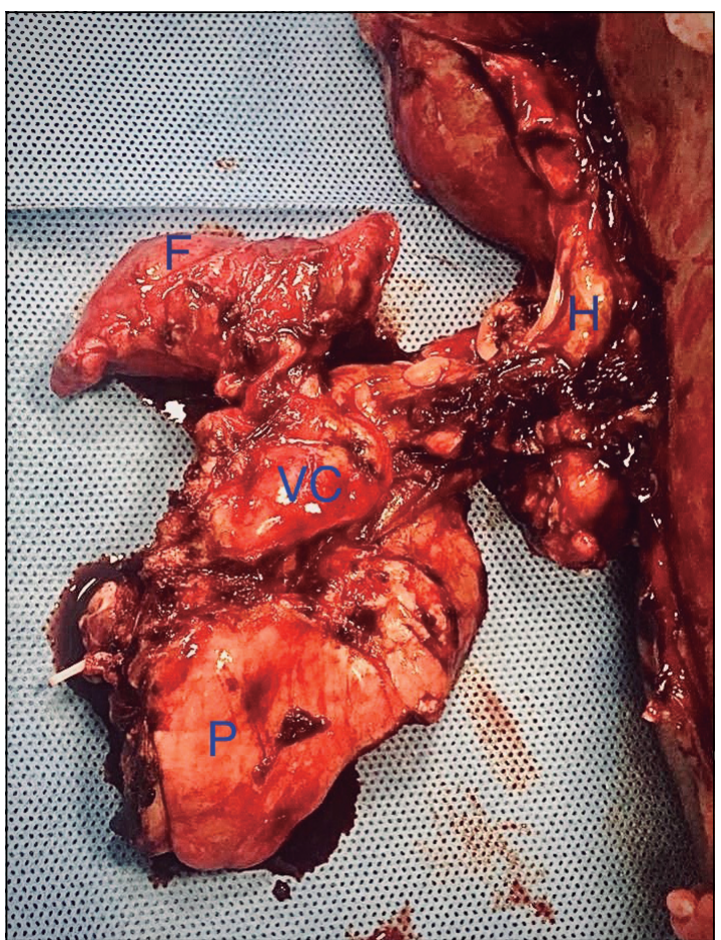

Figura 4. Pieza operatoria donde se observa: segmento de fondo gástrico $(F)$, várices gástricas con cianoacrilato en su interior $(\mathrm{VC})$, hilio esplénico $(\mathrm{H})$ y cola de páncreas $(\mathrm{P})$. 
de su hemoglobina y albuminemia, por ello se decidió continuar por 4 semanas más (6 en total) con ese antimicrobiano.

A 6 meses posterior a la cirugía, el paciente permanece afebril, ya sin tratamiento antibiótico y con colecciones abdominales mínimas en estudio imágenológico. Se diagnosticó un hepatocarcinoma en el segmento lateral, el cual fue tratado exitosamente con radiofrecuencia percutánea y se encuentra en estudio para trasplante hepático.

\section{Discusión}

El hígado es un órgano con múltiples funciones, siendo una de ellas participar en la modulación del sistema inmune a través de diversas formas. Por ello, los pacientes con cirrosis presentan mayor riesgo de desarrollar infecciones, tanto infecciones comunes, como aquellas relacionadas con la fisiopatología de su enfermedad: peritonitis bacteriana espontánea (PBE), bacteriemias recurrentes e infecciones respiratorias, entre otras. En cuanto a las bacteriemias recurrentes, estas pueden ser espontáneas o en relación a procedimientos terapéuticos invasivos, tales como la quimioembolización arterial transcatéter ${ }^{3}$ y la escleroterapia ${ }^{4}$.

En la literatura se describe que los riesgos de bacteriemias transitorias en pacientes cirróticos posterior a la escleroterapia de emergencia o electiva son diferentes ${ }^{6}$, alcanzando valores que oscilan entre $20 \%$ y $70 \%$ para aquellas que son de emergencia y entre 0 y $27 \%$ para las electivas ${ }^{3-5}$.

Sin embargo, la bacteriemia recurrente con repercusión clínica, tal como el síndrome febril prolongado, es una complicación rara posterior a la escleroterapia. Randi et al. ${ }^{6}$ reportan solo 7 casos publicados en la literatura.

En el caso de nuestro paciente, él evolucionó con bacteriemias recurrentes posterior al tratamiento profiláctico de una várice gástrica con cianocrilato.

Dada la rareza del diagnóstico de bacteriemias recurrentes con impacto clínico posterior al tratamiento con cianocrilato, este se debe considerar un diagnóstico de exclusión y se deben investigar otras causas más comunes, como la peritonitis bacteriana espontánea $\mathrm{u}$ otros focos infecciosos. En este sentido, herramientas como el PET-CT con FDG, son útiles en el diagnóstico de un tapón de cianoacrilato infectado ${ }^{7}$. En nuestro caso, este estudio imagenólogico fue central para poder plantear una alternativa quirúrgica como tratamiento. El aumento del metabolismo en el área puede ser normal hasta 2 semanas después de la escleroterapia, pero no después del mes.

En solo uno de los casos reportados por Randi et al. ${ }^{6}$ se realizó manejo quirúrgico resecando el foco infeccioso. Todos los demás fueron manejados con tratamiento antibiótico prolongado $y$ algunos quedaron con profilaxis antibiótica indefinida.

El tratamiento de elección para controlar esta infección son los antibióticos, dado que, además, la mayoría son pacientes cirróticos en los que la morbimortalidad perioperatoria es alta ${ }^{8,10}$. El tratamiento quirúrgico es excepcional y puede ser considerado frente al fracaso del tratamiento antibiótico y en pacientes muy seleccionados, como en el caso que presentamos que permitió la resolución definitiva del síndrome febril.

En el caso expuesto, el paciente, pese a estar con tratamiento antibiótico de amplio espectro y ajuste según hemocultivos, presentaba recurrentemente fiebre y calofríos con repercusión hemodinámica, algunos de ellos con bacteriemias por Escherichia coli. Por tanto, frente a casos con evolución clínica tórpida pese a tratamientos adecuados, que tengan un daño hepático crónico con buena función hepática (Child $\mathrm{A}, \mathrm{o} B$ sin ascitis crónica) y con previa evaluación multidisciplinaria parece prudente plantear una resolución quirúrgica.

\section{Conclusión}

La bacteriemia recurrente con repercusión clínica posterior a la aplicación de cianocrilato a várices gástricas es poco frecuente. Su enfrentamiento inicial debe descartar cualquier otro foco infeccioso que pueda explicar el cuadro. En casos con mala respuesta al tratamiento médico y con evaluación multidisciplinaria parece razonable plantear una resolución quirúrgica previo apoyo diagnóstico con métodos como el PET-CT con FDG.

\section{Referencias}

1. Al-Hillawi L, Wong T, Tritto G, Berry P. Pitfalls in histoacryl glue injection therapy for oesophageal, gastric 
and ectopic varices: A review. World J Gastrointest Surg 2016; 8 (11): 729.

2. Jun C, Kim K, Yoon J, Koh H, Choi W, Cho K et al. Clinical outcomes of gastric variceal obliteration usingN-butyl-2-cyanoacrylate in patients with acute gastric variceal hemorrhage. Korean J Intern Med 2014; 29 (4): 437.

3. Castells A, Bruix J, Ayuso C, Brú C, Montayà X, Boix $\mathrm{L}$, et al. Transarterial embolization for hepatocellular carcinoma. Antibiotic prophylaxis and clinical meaning of postembolization fever. J Hepatol 1995; 22 (4): 410-5.

4. Ho H, Zuckerman M, Wassem C. A prospective controlled study of the risk of bacteremia in emergency sclerotherapy of esophageal varices. Gastroenterology 1991; 101 (6): 1642-8.

5. Rerknimitr R, Chanyaswad J, Kongkam P, Kullavanijaya P. Risk of bacteremia in bleeding and nonbleeding gastric varices after endoscopic injection of cyanoacrylate. Endoscopy 2008; 40 (8): 644-9.

6. Randi BA, Ninomiya DA, Nicodemo EL, Lopes BC, Cançado ER, Levin AS. Recurrent bacteremia after injection of N-butyl-2-cyanoacrylate for treatment of bleeding gastric varices: a case report and review of the literature. BMC Res Notes 2015; 8: 692.

7. Joo K, Hyun I, Baek J, Chung M, Lee J. Detection of an Infected N-butyl-2-cyanoacrylate Plug by F-18 FDG PET/CT Scan in a Patient Who Received Endoscopic Intervention for Gastric Variceal Bleeding. Infect Chemother 2013; 45 (2): 230.

8. Neeff H, Mariaskin D, Spangenberg H, Hopt U, Makowiec F. Perioperative Mortality After Non-hepatic General Surgery in Patients with Liver Cirrhosis: an Analysis of 138 Operations in the 2000s Using Child and MELD Scores. J Gastrointest Surg 2010; 15 (1): $1-11$.

9. Kulkarni SG, Parikh SS, Dhawan PS, Chachad H, Jambavalikar MB, Koppikar GV, et al. High frequency of bacteremia with endoscopic treatment of esophageal varices in advanced cirrhosis. Indian J Gastroenterol 1999; 18: 143-5.

10. Wahl P, Lammer F, Conen D, Schlumpf R, Bock A. Septic complications after injection of N-butyl-2-cyanoacrylate: report of two cases and review. Gastrointest Endosc 2004; 59 (7): 911-6. 